\title{
THE ORTHOGONAL PROJECTION MATRIX FOR THE UNBALANCED FACTORIAL DESIGN
}

\author{
Byung Chun Kim* and Jong Tae Park*
}

\begin{abstract}
The design matrix $X$ for an unbalanced factorial design can be expressed as a product of two matrices $T$ and $X_{o}$, where $T$ is replication matrix and $X_{o}$ is the corresponding balanced design matrix. We suggest an efficient method for computing the orthogonal projection matrix $P_{X}=X\left(X^{\prime} X\right)^{-} X^{\prime}$ for an unbalanced model $\mathbf{y}=X \boldsymbol{\beta}+\mathbf{e}$ using the nonzero eigenvalues and eigenvectors of $X_{o}^{\prime} X_{o}$. Also we can easily find a regular rule for the nonzero eigenvalues and the corresponding eigenvectors.
\end{abstract}

\section{Introduction}

We consider the model

$$
\mathbf{y}=X \boldsymbol{\beta}+\mathbf{e}
$$

where $\mathbf{y}$ is an $N \times 1$ vector of observations, $\boldsymbol{\beta}$ is a $p \times 1$ vector of unknown parameters, $X$ is an $N \times p$ design matrix of 0 's and 1's $(N \geq p)$, and $\mathbf{e}$ is a vector of random error terms. In general, when each cell does not contain the same number of observations or when there are some empty cells, the model is called an unbalanced model. The analysis of this case is not easy and requires much computation. To avoid this difficulty, one needs to easily find the orthogonal projection matrix that is necessary for analyzing the model. The total sum of squares for (1.1) can be decomposed into

$$
\mathbf{y}^{\prime} \mathbf{y}=\mathbf{y}^{\prime} P_{X} \mathbf{y}+\mathbf{y}^{\prime}\left(I-P_{X}\right) \mathbf{y}
$$

where $P_{X}=X\left(X^{\prime} X\right)^{-} X^{\prime}$ and $\left(X^{\prime} X\right)^{-}$is a generalized inverse of $X^{\prime} X$. In view of (1.2) $P_{X}$ is important to compute various sums of squares in the analysis of variance.

The matrix $X\left(X^{\prime} X\right)^{-} X$, denoted by $P_{X}$, is called the orthogonal projection matrix and is unique for any choice of generalized inverse of $X^{\prime} X$. Because of $P_{X}=X X^{+}$where $X^{+}$ is the Moore-Penrose inverse of a matrix $X$, it is useful to find the Moore-Penrose inverse of design matrix in order to compute the orthogonal projection matrix. There are many methods to compute the Moore-Penrose inverse of a matrix. Shinozaki et al.(1972a, 1972b) surveyed the methods for computing the Moore-Penrose inverse into two subjects: direct methods and iterative methods. In order to find the Moore-Penrose inverse of an arbitrary matrix, these methods are very useful. But in most cases the design matrix has the special form, actually the design matrix consists of regular arrangement of 0 's and 1's. Hence we can find $X^{+}$using the Moore-Penrose conditions without using general computational methods. Kim and Sunwoo(1989) found the explicit form of the Moore-Penrose inverse of design

\footnotetext{
*Korea Advanced Institute of Science and Technology, Taejon, Korea.

Key words: orthogonal projection matrix; Moore-Penrose inverse; design matrix.
} 
matrix for the balanced model with no interactions. Also Kim and Sunwoo(1990) derived the iterative method for computing the Moore-Penrose of design matrix for balanced factorial design with interactions and from this the orthogonal projection matrix is computed. But it is more important to compute $P_{X}$ than $X^{+}$from the point of view of statistics. Also when the number of levels of main effects is large or the model has many main and interaction effects, it requires much computation to find $X^{+}$, especially for an unbalanced model. Because of this difficulty, we shall find $\left(X^{\prime} X\right)^{+}$instead of $X^{+}$in obtaining $P_{X}$. Because $X^{\prime} X$ is a symmetric positive semi-definite and hence it is easy to compute $\left(X^{\prime} X\right)^{+}$using the nonzero eigenvalues and eigenvectors of $X_{o}^{\prime} X_{o}$ for the corresponding balanced model.

We will propose an efficient algorithm for computing $P_{X}$ for an unbalanced model using the nonzero eigenvalues and eigenvectors of $X_{o}^{\prime} X_{o}$ as a direct procedure. In section 2 we derive a concrete form of the orthogonal projection matrix for an unbalanced model from the spectral decomposition of $X_{o}^{\prime} X_{o}$. In section 3 we will find the nonzero eigenvalues of $X_{o}^{\prime} X_{o}$ and the corresponding eigenvectors in cases with and without interaction effects. Also some examples will be given.

\section{Orthogonal projection matrix}

For a given unbalanced linear model $\mathbf{y}=X \boldsymbol{\beta}+\mathbf{e}$, the corresponding balanced model is defined as the model that contains exactly one observation in each cell and design matrix of the corresponding balanced model is denoted by $X_{o}$. It is well known that design matrix $X$ can be expressed as a product $X=T X_{o}$ where $T$ is replication matrix. Also the matrix of cell frequencies $D$ can be obtained as $D=T^{\prime} T$. For example, in a two-way additive model

$$
y_{i j k}=\mu+\alpha_{i}+\beta_{j}+e_{i j k}, \quad i=1, \ldots, a ; j=1, \ldots, b ; k=1, \ldots, n_{i j}
$$

with $a=2, b=2, n_{11}=2, n_{12}=3, n_{21}=n_{22}=1$, we would have

$$
\begin{aligned}
X & =\left[\begin{array}{lllll}
1 & 1 & 0 & 1 & 0 \\
1 & 1 & 0 & 1 & 0 \\
1 & 1 & 0 & 0 & 1 \\
1 & 1 & 0 & 0 & 1 \\
1 & 1 & 0 & 0 & 1 \\
1 & 0 & 1 & 1 & 0 \\
1 & 0 & 1 & 0 & 1
\end{array}\right], \quad X_{o}=\left[\begin{array}{lllll}
1 & 1 & 0 & 1 & 0 \\
1 & 1 & 0 & 0 & 1 \\
1 & 0 & 1 & 1 & 0 \\
1 & 0 & 1 & 0 & 1
\end{array}\right] \\
T & =\left[\begin{array}{llll}
1 & 0 & 0 & 0 \\
1 & 0 & 0 & 0 \\
0 & 1 & 0 & 0 \\
0 & 1 & 0 & 0 \\
0 & 1 & 0 & 0 \\
0 & 0 & 1 & 0 \\
0 & 0 & 0 & 1
\end{array}\right], \quad D=\left[\begin{array}{llll}
2 & 0 & 0 & 0 \\
0 & 3 & 0 & 0 \\
0 & 0 & 1 & 0 \\
0 & 0 & 0 & 1
\end{array}\right] .
\end{aligned}
$$

Put $r=\operatorname{rank}\left(X_{o}\right)$. Let $X_{o}^{\prime} X_{o}=Q_{r} \Lambda_{r} Q_{r}^{\prime}$ be a spectral decomposition of $X_{o}^{\prime} X_{o}$ where $\Lambda_{r}$ is a diagonal matrix of positive eigenvalues of $X_{o}^{\prime} X_{o}$ and $Q_{r}$ is a $p \times r$ matrix whose columns are orthonormalized eigenvectors corresponding to positive eigenvalues.

The following lemma gives us the useful facts in obtaining the simple form of the orthogonal projection matrix for an unbalanced model. 
Lemma 2.1. Letting $K_{r}=\Lambda_{r}^{-1 / 2} Q_{r}^{\prime} X^{\prime} X Q_{r} \Lambda_{r}^{-1 / 2}$

$$
\begin{aligned}
X^{\prime} X & =X_{o}^{\prime} D X_{o}=Q_{r} \Lambda_{r}^{1 / 2} K_{r} \Lambda_{r}^{1 / 2} Q_{r}^{\prime}, \\
\left(X^{\prime} X\right)^{+} & =\left(X_{o}^{\prime} D X_{o}\right)^{+}=\left(Q_{r} \Lambda_{r}^{1 / 2} K_{r} \Lambda_{r}^{1 / 2} Q_{r}^{\prime}\right)^{+}=Q_{r} \Lambda_{r}^{-1 / 2} K_{r}^{-1} \Lambda_{r}^{-1 / 2} Q_{r}^{\prime} .
\end{aligned}
$$

Proof. Since $X_{o}^{\prime} X_{o}$ is symmetric positive semi-definite, by spectral decomposition theorem we can write $X_{o}^{\prime} X_{o}=Q \Lambda Q^{\prime}$, where $\Lambda=\operatorname{diag}\left(\lambda_{1}, \ldots, \lambda_{r}, 0, \ldots, 0\right)$ and $Q=\left[Q_{r} Q_{o}\right]$ is an orthogonal matrix partitioned so that $Q_{r}$ is a $p \times r$ matrix. Then $Q_{o}^{\prime} Q_{o}=I_{p-r}, Q_{r}^{\prime} Q_{r}=I_{r}$ and $Q_{o} Q_{o}^{\prime}+Q_{r} Q_{r}^{\prime}=I_{p}$ since $Q$ is orthogonal matrix. From $K_{r}=\Lambda_{r}^{-1 / 2} Q_{r}^{\prime} X^{\prime} X Q_{r} \Lambda_{r}^{-1 / 2}$,

$$
Q_{r} \Lambda_{r}^{1 / 2} K_{r} \Lambda_{r}^{1 / 2} Q_{r}^{\prime}=X_{o}^{\prime} D X_{o}=X^{\prime} X
$$

since $Q_{o}^{\prime} X_{o}^{\prime} X_{o} Q_{o}=0$ and also $X_{o} Q_{o}=0$. Next the proof of $(2.2)$ can be easily verified.

Using these properties we can find the simple form of the orthogonal projection matrix for an unbalanced model. The form is as follows.

Theorem 2.1. For matrices defined above

$$
P_{X}=T Z K_{r}^{-1} Z^{\prime} T^{\prime}
$$

where $Z=X_{o} Q_{r} \Lambda_{r}^{-1 / 2}$ and $K_{r}=Z^{\prime} D Z$.

In general the size of $P_{X}$ is very large. But as is shown in (2.3) the prime part of computations are accomplished by handling matrices with smaller size. The remaining computations are merely matrix multiplications. Since $K_{r}$ is an $r \times r$ symmetric positive definite, by Cholesky decomposition, we can write $K_{r}=L L^{\prime}$ where $L$ is a lower triangular matrix with positive diagonal elements. Furthermore, $K_{r}^{-1}=\left(L^{-1}\right)^{\prime} L^{-1}$. Hence it is important to find $Z$ in order to compute $P_{X}$ because of $K_{r}=Z^{\prime} D Z$. Also it is interesting to note that $Z Z^{\prime}=P_{X_{\circ}}$ and $Z^{\prime} Z=I_{r}$ hold. A concrete form of $Z=X_{o} Q_{r} \Lambda_{r}^{-1 / 2}$ is described in the next section. If $Z$ is known for each classification model, it is useful to compute the reduction sum of squares. For example, partitioning main effects $\boldsymbol{\alpha}$ as $\boldsymbol{\alpha}^{\prime}=\left[\boldsymbol{\alpha}_{1}^{\prime}, \boldsymbol{\alpha}_{2}^{\prime}, \ldots, \boldsymbol{\alpha}_{k}^{\prime}\right]$ for $k$-way factorial design, Type II sum of squares is as follows:

$$
\begin{aligned}
& R\left(\boldsymbol{\alpha}_{i} \mid \mu, \boldsymbol{\alpha}_{1}, \ldots, \boldsymbol{\alpha}_{i-1}, \boldsymbol{\alpha}_{i+1}, \ldots, \boldsymbol{\alpha}_{k}\right) \\
= & R\left(\mu, \boldsymbol{\alpha}_{1}, \ldots, \boldsymbol{\alpha}_{k}\right)-R\left(\mu, \boldsymbol{\alpha}_{1}, \ldots, \boldsymbol{\alpha}_{i-1}, \boldsymbol{\alpha}_{i+1}, \ldots, \boldsymbol{\alpha}_{k}\right) \\
= & \mathbf{y}^{\prime} P_{X} \mathbf{y}-\mathbf{y}^{\prime} P_{X_{-i}} \mathbf{y}
\end{aligned}
$$

where $X_{-i}$ is the design matrix for $(k-1)$-way factorial design that is removing $i$ th main effect from the original model.

\section{Eigenvalues and eigenvectors}

Let $X_{o}$ be the corresponding balanced design matrix. With a help of matrix algebra we know that positive eigenvalues and eigenvectors of $X_{o}^{\prime} X_{o}$ depend on only the number of levels of main effects by a regular rule. From this we can find an explicit form of the corresponding eigenvectors. For the regular rule of positive eigenvalues and eigenvectors of $X_{o}^{\prime} X_{o}$ we will deal with two cases: with and without interaction effects.

We shall use boldface lower case letters to denote column vectors and the following notation: $\mathbf{1}_{m}$ is an $\boldsymbol{m}$-dimensional column vector of unities and $\mathbf{0}_{m}$ is an $m$-dimensional column vector of zeros. 


\subsection{Case without interaction effects}

Since $X_{o}$ has special form of 0 's and 1's, positive eigenvalues of $X_{o}^{\prime} X_{o}$ and the corresponding eigenvectors can be easily found as shown in the following theorem.

Theorem 3.1.1. Let $X_{o}=\left[1_{m} X_{1} \cdots X_{k}\right]$ be the corresponding balanced design matrix for the $k$-way model

$$
\mathbf{y}=\mathbf{1}_{m} \mu+X_{1} \boldsymbol{\alpha}_{1}+\cdots+X_{k} \boldsymbol{\alpha}_{k}+\mathbf{e}
$$

where $m=\prod_{j=1}^{k} m_{j}$ and $m_{j}$ is the number of levels of $j$ th main effect. Then for positive eigenvalues $\lambda_{i}$ 's of $X_{o}^{\prime} X_{o}$

$$
\mathbf{u}_{0}=\left[m \frac{m}{m_{1}} 1_{m_{1}}^{\prime} \cdots \frac{m}{m_{k}} 1_{m_{k}}^{\prime}\right]^{\prime}
$$

is the eigenvector corresponding to $\lambda_{0}=m+\sum_{j=1}^{k}\left(m / m_{j}\right)$; for $j=1, \ldots, k$

$$
\mathbf{u}_{i}=\left[\begin{array}{ll}
0 & \mathbf{0}_{m_{1}}^{\prime}
\end{array} \cdots\left(\mathbf{e}_{1}^{(j)}-\mathbf{e}_{i+1}^{(j)}\right)^{\prime} \cdots \mathbf{0}_{m_{k}}^{\prime}\right]^{\prime}, \quad i=1, \ldots, m_{j}-1
$$

are the eigenvectors corresponding to $\lambda_{j}=m / m_{j}$, where $\mathbf{e}_{i}^{(j)}$ is an $m_{j} \times 1$ vector having 1 as $i$-th entry and zero elsewhere, i.e., $\mathbf{e}_{i}^{(j)}=\left(\begin{array}{lllll}0 & \cdots & 1 & 0 & \cdots\end{array}\right)^{\prime}$.

Proof. Since the incidence matrices $X_{i}$ 's can be expressed using Kronecker products of identity matrix and vectors with all elements equal to unity, that is,

$$
X_{i}=\mathbf{1}_{m_{1}} \otimes \cdots \otimes I_{m_{i}} \otimes \cdots \otimes \mathbf{1}_{m_{k}}
$$

it is easy to see that the following relations hold:

$$
\begin{gathered}
X_{i}^{\prime} X_{i}=\frac{m}{m_{i}} I_{m_{i}}, \quad X_{i}^{\prime} \mathbf{1}_{m}=\frac{m}{m_{i}} \mathbf{1}_{m_{i}}, \quad X_{i} \mathbf{1}_{m_{i}}=\mathbf{1}_{m} \quad \text { for } \quad i=1, \cdots, k \\
X_{i}^{\prime} X_{j}=\frac{m}{m_{i} m_{j}} J_{m_{i} \times m_{j}} \text { for } \quad i \neq j
\end{gathered}
$$

where $J_{m_{i} \times m_{j}}=\mathbf{1}_{m_{i}} \mathbf{1}_{m_{j}}^{\prime}$. Then

$$
X_{o}^{\prime} X_{o} \mathbf{u}_{0}=\left[\begin{array}{c}
m^{2}+\sum_{j=1}^{k} \lambda_{j} \mathbf{1}_{m}^{\prime} X_{j} \mathbf{1}_{m_{j}} \\
m X_{1}^{\prime} \mathbf{1}_{m}+\sum_{j=1}^{k} \lambda_{j} X_{1}^{\prime} X_{j} \mathbf{1}_{m_{j}} \\
\vdots \\
m X_{k}^{\prime} \mathbf{1}_{m}+\sum_{j=1}^{k} \lambda_{j} X_{k}^{\prime} X_{j} \mathbf{1}_{m_{j}}
\end{array}\right]=\left(m+\sum_{j=1}^{k} \lambda_{j}\right)\left[\begin{array}{c}
m \\
\lambda_{1} \mathbf{1}_{m_{1}} \\
\vdots \\
\lambda_{k} \mathbf{1}_{m_{k}}
\end{array}\right]
$$

Hence $\mathbf{u}_{0}$ is the eigenvector of $X_{o}^{\prime} X_{o}$ corresponding to $\lambda_{0}=m+\sum_{j=1}^{k} \lambda_{j}$. Similarly, the remaining part follows from the relations described above.

As special cases of theorem 3.1.1, positive eigenvalues of $X_{o}^{\prime} X_{0}$ for two- and threeway factorial designs are as follows. The numbers in brackets are multiplicities of the corresponding eigenvalues.

(i) Two-way model: setting $m_{1}=a, m_{2}=b$

$$
\text { eigenvalues : } a b+b+a[1], b[a-1], a[b-1]
$$


Orthogonal projection matrix for the unbalanced factorial design

(ii) Three-way model: setting $m_{1}=a, m_{2}=b, m_{3}=c$

eigenvalues : $a b c+b c+a c+a b[1], b c[a-1], a c[b-1], a b[c-1]$

By theorem 3.1.1 we can construct $Q_{r}$ for the $k$-way model from Gram-Schmidt orthogonalization. Using $Q_{r}$ and $\Lambda_{r}$ we also can easily find $Z=X_{o} Q_{r} \Lambda_{r}^{-1 / 2}$. Put $Z=\left[\begin{array}{lll}\mathbf{z}_{0} & Z_{1} \cdots Z_{k}\end{array}\right]$ where $Z_{j}=\left[\mathbf{z}_{1} \cdots \mathbf{z}_{m_{j-1}}\right], j=1, \ldots, k$. Then

$$
\begin{aligned}
\mathbf{z}_{0} & =\frac{1}{\sqrt{m}} \mathbf{1}_{m} \\
\mathbf{z}_{i}^{(j)} & =\frac{\sqrt{m_{j}}}{\sqrt{m} \sqrt{i(i+1)}}\left[\mathbf{1}_{m_{1}} \otimes \cdots \otimes\left(\sum_{t=1}^{i} \mathbf{e}_{t}^{(j)}-i \mathbf{e}_{i+1}^{(j)}\right) \otimes \cdots \otimes \mathbf{1}_{m_{k}}\right]
\end{aligned}
$$

for all $j=1, \ldots, k$ and $i=1, \ldots, m_{j}-1$.

From (3.1.4) and (3.1.5) we know that the computation of $Z$ requires only the number of levels of main effects. Also note that $\sum_{t=1}^{i} \mathbf{e}_{t}^{(j)}-i \mathbf{e}_{i+1}^{(j)}$ in (3.1.5) is an $m_{j} \times 1$ vector having 1 as first through $i$-th components, $-i$ as $i+1$-th component and zero elsewhere, that is, $\sum_{t=1}^{i} \mathbf{e}_{t}^{(j)}-i \mathbf{e}_{i+1}^{(j)}=(1 \cdots 1-i 0 \cdots 0)^{\prime}$.

We introduce a simple example to find the orthogonal projection matrix $P_{X}$ for the three-way model

$$
\mathbf{y}=\mathbf{1}_{m} \mu+X_{1} \boldsymbol{\alpha}+X_{2} \boldsymbol{\beta}+X_{3} \boldsymbol{\gamma}+\mathbf{e}
$$

with $m_{1}=m_{2}=m_{3}=2$.

Example 1. For model (3.1.6) with cell frequencies $n_{111}=n_{122}=n_{212}=n_{221}=1$, $n_{112}=n_{121}=n_{211}=n_{222}=2$, we would get

$$
X=\left[\begin{array}{lllllll}
1 & 1 & 0 & 1 & 0 & 1 & 0 \\
1 & 1 & 0 & 1 & 0 & 0 & 1 \\
1 & 1 & 0 & 1 & 0 & 0 & 1 \\
1 & 1 & 0 & 0 & 1 & 1 & 0 \\
1 & 1 & 0 & 0 & 1 & 1 & 0 \\
1 & 1 & 0 & 0 & 1 & 0 & 1 \\
1 & 0 & 1 & 1 & 0 & 1 & 0 \\
1 & 0 & 1 & 1 & 0 & 1 & 0 \\
1 & 0 & 1 & 1 & 0 & 0 & 1 \\
1 & 0 & 1 & 0 & 1 & 1 & 0 \\
1 & 0 & 1 & 0 & 1 & 0 & 1 \\
1 & 0 & 1 & 0 & 1 & 0 & 1
\end{array}\right], \quad X_{o}=\left[\begin{array}{lllllll}
1 & 1 & 0 & 1 & 0 & 1 & 0 \\
1 & 1 & 0 & 1 & 0 & 0 & 1 \\
1 & 1 & 0 & 0 & 1 & 1 & 0 \\
1 & 1 & 0 & 0 & 1 & 0 & 1 \\
1 & 0 & 1 & 1 & 0 & 1 & 0 \\
1 & 0 & 1 & 1 & 0 & 0 & 1 \\
1 & 0 & 1 & 0 & 1 & 1 & 0 \\
1 & 0 & 1 & 0 & 1 & 0 & 1
\end{array}\right],
$$




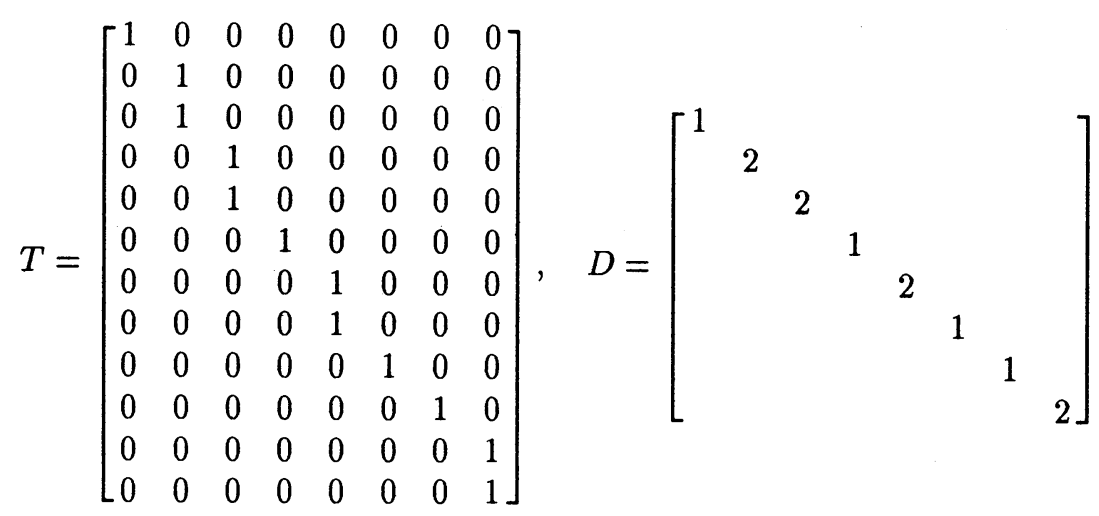

Then from (3.1.4) and (3.1.5) appropriate matrices are as follows:

$$
\begin{aligned}
& \Lambda_{r}=\left[\begin{array}{llll}
20 & & & \\
& 4 & & \\
& & 4 & \\
& & & 4
\end{array}\right], \quad Z=\frac{1}{2 \sqrt{2}}\left[\begin{array}{rrrr}
1 & 1 & 1 & 1 \\
1 & 1 & 1 & -1 \\
1 & 1 & -1 & 1 \\
1 & 1 & -1 & -1 \\
1 & -1 & 1 & 1 \\
1 & -1 & 1 & -1 \\
1 & -1 & -1 & 1 \\
1 & -1 & -1 & -1
\end{array}\right], \\
& K_{r}=\frac{3}{2} I_{4}
\end{aligned}
$$

Hence from (2.3) $P_{X}$ becomes

$$
P_{X}=\frac{1}{6}\left[\begin{array}{rrrrrrrrrrrr}
2 & 1 & 1 & 1 & 1 & 0 & 1 & 1 & 0 & 0 & -1 & -1 \\
1 & 2 & 2 & 0 & 0 & 1 & 0 & 0 & 1 & -1 & 0 & 0 \\
1 & 2 & 2 & 0 & 0 & 1 & 0 & 0 & 1 & -1 & 0 & 0 \\
1 & 0 & 0 & 2 & 2 & 1 & 0 & 0 & -1 & 1 & 0 & 0 \\
1 & 0 & 0 & 2 & 2 & 1 & 0 & 0 & -1 & 1 & 0 & 0 \\
0 & 1 & 1 & 1 & 1 & 2 & -1 & -1 & 0 & 0 & 1 & 1 \\
1 & 0 & 0 & 0 & 0 & -1 & 2 & 2 & 1 & 1 & 0 & 0 \\
1 & 0 & 0 & 0 & 0 & -1 & 2 & 2 & 1 & 1 & 0 & 0 \\
0 & 1 & 1 & -1 & -1 & 0 & 1 & 1 & 2 & 0 & 1 & 1 \\
0 & -1 & -1 & 1 & 1 & 0 & 1 & 1 & 0 & 2 & 1 & 1 \\
-1 & 0 & 0 & 0 & 0 & 1 & 0 & 0 & 1 & 1 & 2 & 2 \\
-1 & 0 & 0 & 0 & 0 & 1 & 0 & 0 & 1 & 1 & 2 & 2
\end{array}\right]
$$

\subsection{Case with interaction effects}

Before we describe the general procedure we consider the representations of the model equation. We denote the incidence matrix of interaction effects between $i$-th and $j$-th factor by $V_{\{i, j\}}$ and between $i$-th, $j$-th, and $k$-th factor by $V_{\{i, j, k\}}$, and so on. For the purpose of explaining our results consider the set of integers $T=\{1,2, \ldots, k\}$ and let $S_{j}$ be a subset of $T$ which contains at least two elements of $T$ such that $n\left(S_{1}\right) \leq n\left(S_{2}\right) \leq \ldots \leq n\left(S_{c}\right)$, where $n\left(S_{j}\right)$ is the size of set $S_{j}, j=1,2, \ldots, c$ for some integer $c . V_{j}=V_{S_{j}}$ denotes the 
incidence matrix of interaction effects and their indices are members of $S_{j}$. It is well known that for $k$-way factorial design the incidence matrices can be expressed using Kronecker products(Rogers(1984)):

$$
\begin{aligned}
& V_{j}=V_{S_{j}}=\otimes_{i=1}^{k} A_{i} \\
& \text { where } A_{i}= \begin{cases}\mathbf{1}_{m_{i}} & \text { if } i \notin S_{j}, \\
I_{m_{i}} & \text { if } i \in S_{j} .\end{cases}
\end{aligned}
$$

Now let $X_{o}=\left[1_{m} U_{o} V_{o}\right]$ be the balanced design matrix for the model with $k$ main effects and $c$ interaction effects

$$
\mathbf{y}=\mathbf{1}_{m} \boldsymbol{\mu}+U_{o} \boldsymbol{\alpha}+V_{o} \boldsymbol{\beta}+\mathbf{e}
$$

where $U_{o}$ is the incidence matrix of main effects and $V_{o}=\left[\begin{array}{llll}V_{1} & V_{2} & \ldots & V_{c}\end{array}\right], V_{j}$ is an $m \times d_{j}$ matrix of known values $\left(d_{j}=\prod_{s \in S_{j}} m_{s}\right)$, and other terms are defined as usual. Then for positive eigenvalues and eigenvectors of $X_{o}^{\prime} X_{o}$ we can obtain the following theorem based on the case without interaction effects.

Theorem 3.2.1. For model (3.2.3) positive eigenvalues of $X_{o}^{\prime} X_{o}$ are as follows:

(i)

$$
\begin{gathered}
\lambda=\lambda_{0}^{*}[1], \quad \lambda_{i}^{*}\left[m_{i}-1\right](i=1, \ldots, k), \quad \lambda_{k+j}^{*}\left[\prod_{s \in S_{j}}\left(m_{s}-1\right)\right](j=1, \ldots, c) \\
\lambda_{0}^{*}=\lambda_{0}+\sum_{j=1}^{c} \frac{m}{d_{j}}, \quad \text { where } \lambda_{0}=m+\sum_{i=1}^{k} \frac{m}{m_{i}}, \\
\lambda_{i}^{*}=\lambda_{i}+\sum_{j=1}^{c} h_{j}, \quad i=1, \ldots, k, \\
\text { where } \quad \lambda_{i}=\frac{m}{m_{i}} \quad \text { and } \quad h_{j}= \begin{cases}m / d_{j} & \text { if } i \in S_{j} \\
0 & \text { if } i \notin S_{j},\end{cases} \\
\lambda_{k+j}^{*}=\lambda_{k+j}+\sum_{n=j+1}^{c} t_{n}, \quad j=1, \ldots, c, \quad \text { if } S_{j} \subset S_{n} \\
\text { where } \quad \lambda_{k+j}=\frac{m}{d_{j}} \quad \text { and } t_{n}= \begin{cases}m / d_{n} & \text { if } S_{j} \not \subset S_{n} . \\
0 & \end{cases}
\end{gathered}
$$

The numbers in brackets are multiplicities of the corresponding eigenvalues.

(ii)

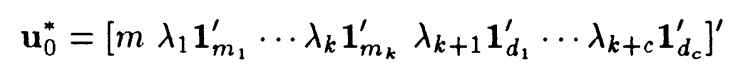

is the eigenvector of $X_{o}^{\prime} X_{o}$ corresponding to $\lambda=\lambda_{0}^{*}$. And for $i=1, \ldots, k$

$$
\mathbf{u}_{l}^{*}=\left[\begin{array}{ll}
0 & \mathbf{0}_{m_{1}}^{\prime} \cdots \lambda_{i}\left(\mathbf{e}_{1 i}-\mathbf{e}_{l+1 i}\right)_{m_{i}}^{\prime} \cdots \mathbf{0}_{m_{k}}^{\prime} \lambda_{k+1} \mathbf{p}_{1}^{\prime} \cdots \lambda_{k+c} \mathbf{p}_{c}^{\prime}
\end{array}\right]^{\prime}, \quad l=1, \ldots, m_{i}-1
$$

are the eigenvectors of $X_{o}^{\prime} X_{o}$ corresponding to $\lambda=\lambda_{i}^{*}$, where

$$
\mathbf{p}_{j}=\left\{\begin{array}{ll}
\otimes_{s \in S_{j}} \mathbf{b}_{s} & \text { if } i \in S_{j} \\
\mathbf{0}_{d_{j}} & \text { if } i \notin S_{j}
\end{array}, \quad \mathbf{b}_{s}=\left\{\begin{array}{ll}
\mathbf{e}_{1}^{(i)}-\mathbf{e}_{l+1}^{(i)} & \text { if } s=i \\
\mathbf{1}_{m}, & \text { if } s \neq i
\end{array} .\right.\right.
$$


(iii) for $j=1, \ldots, c$

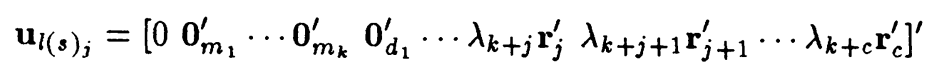

are the eigenvectors of $X_{o}^{\prime} X_{o}$ corresponding to $\lambda=\lambda_{k+j}^{*}$, where the index notation $l(s)_{j}$ denotes a possible combination of $\left\{1, \ldots, m_{s}-1\right\}$ for each $s \in S_{j}$, and

$$
\mathbf{r}_{j}=\otimes_{s \in S_{j}}\left(\mathbf{e}_{1}^{(s)}-\mathbf{e}_{l(s)+1}^{(s)}\right)
$$

and for $n=j+1, \ldots, c$

$$
\mathbf{r}_{n}=\left\{\begin{array}{ll}
\otimes_{s \in S_{n}} \mathbf{c}_{s} & \text { if } S_{j} \subset S_{n} \\
\mathbf{0}_{d_{n}} & \text { if } S_{j} \not \subset S_{n}
\end{array}, \quad \mathbf{c}_{s}=\left\{\begin{array}{ll}
\mathbf{e}_{1}^{(s)}-\mathbf{e}_{l(s)+1}^{(s)} & \text { if } s \in S_{j} \\
\mathbf{1}_{m_{s}} & \text { if } s \notin S_{j}
\end{array} .\right.\right.
$$

Proof. Similarlly as in theorem 3.1.1, the theorem can be easily proved.

As example for (c), when a three-way model with $\boldsymbol{\alpha} \boldsymbol{\beta}, \boldsymbol{\alpha} \boldsymbol{\beta} \boldsymbol{\gamma}$ interaction effects has $m_{1}=2, m_{2}=m_{3}=3$, it does $S_{1}=\{1,2\}, S_{2}=\{1,2,3\}$, and $\left\{l(s)_{1} \mid s \in S_{1}\right\}=\{11,12\}$, $\left\{l(s)_{2} \mid s \in S_{2}\right\}=\{111,112,121,122\}$.

From theorem 3.2.1 we can find a regular rule that changes the eigenvalues for the resulting model when the interaction effects are added the original model. Also this rule can be applied to the corresponding eigenvectors. For example, positive eigenvalues correspond to each effect one by one:

$$
\begin{aligned}
\mu & \rightarrow \lambda_{o}=m+\sum_{i=1}^{k}\left(m / m_{i}\right) \\
\alpha_{i} & \rightarrow \lambda_{i}=m / m_{i} \\
\beta_{j} & \rightarrow \lambda_{k+j}=m / d_{j} .
\end{aligned}
$$

Then the new eigenvalues $\lambda_{i}^{*}$ and $\lambda_{k+j}^{*}$ are changed by adding an eigenvalue corresponding to the effect whenever there is interaction effect containing $i$ th main effect and $j$ th interaction effect, respectively. Also $\lambda_{o}^{*}=\lambda_{o}+\sum_{j=1}^{c} \lambda_{k+j}$.

As in the case without interaction effects, we can easily compute $Z$ by Gram-Schmidt

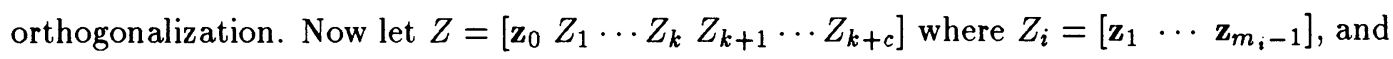
$Z_{k+j}$ is a matrix whose columns are $\mathbf{z}_{l(s)}$ 's. Then for all $i=1, \ldots, k$ and $l=1, \ldots, m_{i}-1$

$$
\begin{aligned}
& \mathbf{z}_{0}=\frac{1}{\sqrt{m}} \mathbf{1}_{m} \\
& \mathbf{z}_{l}=\frac{\sqrt{m_{i}}}{\sqrt{m} \sqrt{l(l+1)}}\left[\mathbf{1}_{m_{1}} \otimes \cdots \otimes\left(\sum_{k=1}^{l} \mathbf{e}_{k}^{(i)}-l \mathbf{e}_{l+1}^{(i)}\right) \otimes \cdots \otimes \mathbf{1}_{m_{k}}\right]
\end{aligned}
$$

and for all $j=1, \ldots, c$ and $l(s)=1, \ldots, m_{s}-1$ for all $s \in S_{j}$

$$
\mathbf{z}_{l(s)_{j}}=\frac{\sqrt{d_{j}}}{\sqrt{m} \sqrt{\sum_{s \in S_{j}} l(s)(l(s)+1)}}\left[\otimes_{i=1}^{k} B_{i}\right]
$$


where

$$
B_{i}= \begin{cases}\sum_{t=1}^{l} \mathbf{e}_{t}^{(i)}-l \mathbf{e}_{l+1}^{(i)} & \text { if } i \in S_{j} \\ \mathbf{1}_{m_{i}} & \text { if } i \notin S_{j}\end{cases}
$$

Example 2. Consider the three-way model with $\boldsymbol{\alpha} \boldsymbol{\beta}$ interaction effect

$$
\mathbf{y}=\mathbf{1}_{m} \boldsymbol{\mu}+X_{1} \boldsymbol{\alpha}+X_{2} \boldsymbol{\beta}+X_{\mathbf{3}} \boldsymbol{\gamma}+V(\boldsymbol{\alpha} \boldsymbol{\beta})+\mathbf{e}
$$

having $m_{1}=m_{2}=m_{3}=2, S_{1}=\{1,2\}, d_{1}=m_{1} m_{2}=4$, and cell frequencies $n_{111}=n_{122}=$ $n_{212}=n_{221}=1, n_{112}=n_{121}=n_{211}=n_{222}=2$.

In this case, the positive eigenvalues of $X_{o}^{\prime} X_{o}$ are as follows:

$$
\lambda_{0}^{*}=22[1], \quad \lambda_{1}^{*}=\lambda_{2}^{*}=6[1], \quad \lambda_{3}^{*}=4[1], \quad \lambda_{4}^{*}=2[1]
$$

where the numbers in brackets are multiplicities of the corresponding eigenvalues.

Also as in example 1 appropriate matrices are given by

$$
Z=\frac{1}{2 \sqrt{2}}\left[\begin{array}{rrrrr}
1 & 1 & 1 & 1 & 1 \\
1 & 1 & 1 & -1 & 1 \\
1 & 1 & -1 & 1 & -1 \\
1 & 1 & -1 & -1 & -1 \\
1 & -1 & 1 & 1 & -1 \\
1 & -1 & 1 & -1 & -1 \\
1 & -1 & -1 & 1 & 1 \\
1 & -1 & -1 & -1 & 1
\end{array}\right], \quad K_{r}=\frac{1}{2}\left[\begin{array}{rrrrr}
3 & 0 & 0 & 0 & 0 \\
0 & 3 & 0 & 0 & 0 \\
0 & 0 & 3 & 0 & 0 \\
0 & 0 & 0 & 3 & -1 \\
0 & 0 & 0 & -1 & 3
\end{array}\right]
$$

from (3.2.10), (3.2.11) and (3.2.12). By (2.3) $P_{X}$ becomes

$$
P_{\boldsymbol{X}}=\frac{1}{24}\left[\begin{array}{rrrrrrrrrrrr}
12 & 6 & 6 & 2 & 2 & -4 & 2 & 2 & -4 & 4 & -2 & -2 \\
6 & 9 & 9 & -1 & -1 & 2 & -1 & -1 & 2 & -2 & 1 & 1 \\
6 & 9 & 9 & -1 & -1 & 2 & -1 & -1 & 2 & -2 & 1 & 1 \\
2 & -1 & -1 & 9 & 9 & 6 & 1 & 1 & -2 & 2 & -1 & -1 \\
2 & -1 & -1 & 9 & 9 & 6 & 1 & 1 & -2 & 2 & -1 & -1 \\
-4 & 2 & 2 & 6 & 6 & 12 & -2 & -2 & 4 & -4 & 2 & 2 \\
2 & -1 & -1 & 1 & 1 & -2 & 9 & 9 & 6 & 2 & -1 & -1 \\
2 & -1 & -1 & 1 & 1 & -2 & 9 & 9 & 6 & 2 & -1 & -1 \\
-4 & 2 & 2 & -2 & -2 & 4 & 6 & 6 & 12 & -4 & 2 & 2 \\
4 & -2 & -2 & 2 & 2 & -4 & 2 & 2 & -4 & 12 & 6 & 6 \\
-2 & 1 & 1 & -1 & -1 & 2 & -1 & -1 & 2 & 6 & 9 & 9 \\
-2 & 1 & 1 & -1 & -1 & 2 & -1 & -1 & 2 & 6 & 9 & 9
\end{array}\right]
$$

\section{Concluding remarks}

From (2.3) we can easily obtain the orthogonal projection matrix for a given unbalanced model by computing the inverse matrix and then multiplying the resulting matrices. Although the notation in theorem 3.1.1 and theorem 3.2.1 seems to be complicated, the computing procedure for $Z$ as shown in (3.1.5), (3.2.11) and (3.2.12) is simple and efficient whether or not there are interactions because the expressions depend only on the number of levels of main effects. By using $Z$ we also can reduce much of the computational effort and computational storage since computing $P_{X}$ relies upon $Z$. It is useful to compute various sums of squares in the analysis of variance. As well, it can be applied to computing $F$-statistic for testing a general linear hypothesis of the form $H: K^{\prime} \boldsymbol{\beta}=\mathbf{m}$. 


\section{KIM and PARK}

\section{Acknowledgements}

The authors wish to thank the referees and the associate editor for their helpful comments.

\section{REFERENCES}

Graybill, F. A.(1969). Introduction to Matrices with Applications in Statistics, Wadworth Publishing Company, Belmont, Califonia.

Kim, B. C. and Sunwoo, H. S.(1989). The Moore-Penrose inverse for the balanced ANOVA models. J. of the Japanese Soc. of Comp. Statist., 2, 1-7.

Kim, B. C. and Sunwoo, H. S.(1990). The M-P inverse for the balanced factorial design with interactions. J. of the Japanese Soc. of Comp. Statist., 3, 25-34.

Rogers, G. S.(1984). Kronecker products in ANOVA - a first step. The Amer. Statist., 38, 197-202.

Searle, S. R.(1982). Matrix Algebra Useful for Statistics, John Wiley and Sons, New York.

Shinozaki, N., Sibuya, M., and Tanabe, K.(1972a). Numerical algorithms for the Moore-Penrose inverse of a matrix: direct methods. Ann. Inst. Statist. Math., 24, 193-203.

Shinozaki, N., Sibuya, M., and Tanabe, K.(1972b). Numerical algorithms for the Moore-Penrose inverse of a matrix: iterative methods. Ann. Inst. Statist. Math., 24, 621-629.

(Received May, 1992; Revised November, 1992) 